\title{
Preoperative circulating FPR and CCF score are promising biomarkers for predicting clinical outcome of stage II-III colorectal cancer patients
}

This article was published in the following Dove Press journal: Cancer Management and Research

\author{
Fan Sun ${ }^{1, *}$ \\ Hong-Xin Peng ${ }^{2, *}$ \\ Qiu-Fang Gao' \\ Shu-Qi Li' \\ Jing Zhang' \\ Qing-Gen Chen' \\ Yu-Huan Jiang' \\ Lei Zhang' \\ Xiao-Zhong Wang \\ Hou-Qun Ying' \\ 'Department of Clinical Laboratory, \\ Jiangxi Province Key Laboratory of \\ Laboratory Medicine, The Second \\ Affiliated Hospital of Nanchang \\ University, Nanchang 330006, Jiangxi, \\ China; ${ }^{2}$ Department of Clinical \\ Laboratory, Nanjing First Hospital, \\ Nanjing Medical University, Nanjing \\ 210006 , Jiangsu, China \\ *These authors contributed equally to \\ this work
}

Correspondence: Hou-Qun Ying; Xiao-Zhong Wang

Department of Clinical Laboratory, Jiangxi Province Key Laboratory of Laboratory Medicine, The Second Affiliated Hospital of Nanchang University, No. I Minde Rd, Nanchang 330006, China

$\mathrm{Tel} / \mathrm{Fax}+8679186300410$

Email yinghouqun2013@163.com; wangxiaozhong@ncu.edu.cn
Introduction: Inflammation and nutrition are considered as two important causes leading to the progression and poor survival of colorectal cancer (CRC). The objective of this study is to investigate the prognostic significance of preoperative albumin-to-fibrinogen ratio (AFR), fibrinogen-to-pre-albumin ratio (FPR), fibrinogen (Fib), albumin (Alb), and pre-albumin (preAlb) in CRC individuals.

Materials and methods: In this study, 3 years' follow-up was carried out in 702 stage I-III resected CRC patients diagnosed between January 2008 and December 2013. The optimal cutoff points and prognostic values of AFR, FPR, Fib, Alb, pre-Alb, and a novel carcinoembryonic antigen (CEA)-carbohydrate antigen 19-9 (CA199)-FPR (CCF) score were assessed by X-tile software, Kaplan-Meier curve, and Cox regression model. We established the CRC prognostic nomogram, and its predictive efficacy was determined by Harrell's concordance index (c-index). Results: Our results showed that high FPR was obviously correlated with poor survival of CRC patients. The prognostic predictive efficacy of CCF score was superior to FPR, CEA, CA199, CEA-CA199 (CCI), and CEA-FPR (CFI) score. Moreover, stage II-III patients harboring high FPR or elevated $C C F$ (score $\geq 1$ ) could benefit from adjuvant chemotherapy, rather than those with low FPR or CCF (score=0). Additionally, the c-index (0.728) of the nomogram containing CCF score was significantly higher than that $(0.626)$ without it $(p<0.01)$.

Conclusion: These findings illustrated that FPR and CCF score were promising biomarkers to predict the prognosis of CRC and to classify the stage II-III patients who could benefit from the adjuvant chemotherapy.

Keywords: inflammation, colorectal cancer, FPR, CCF score, survival

\section{Introduction}

Colorectal cancer (CRC) is one of the most common malignancies and a leading cause in the last decade of cancer-related death worldwide; ${ }^{1,2}$ it is also the fifth most common cancer and the fifth leading cause of death in China. ${ }^{3}$ Although substantial progress had been achieved in decades, $\sim 60 \%$ of $\mathrm{CRC}$ individuals were diagnosed with node or distant metastasis and the 5 years' survival rate was merely $65 \%$ from 2006 to $2012 .{ }^{4}$ Thus, novel, effective, stable, and economical biomarkers may help to improve diagnostic and predictive efficacy of the disease.

It was reported that an estimated $15 \%$ of the cancer-related death was attributed to chronic inflammation, ${ }^{5}$ and accumulating evidence indicated that inflammation was one of the most crucial causes facilitating onset and metastasis of CRC. ${ }^{6}$ Recent studies have found vital roles of inflammation-induced genetic alternation, immune cells, cytokines, and other mediators in each step of colonic tumorigenesis and progression. ${ }^{7-10}$ Moreover, 
the continuous use of low-dose nonsteroidal anti-inflammatory drugs was associated with a decreased risk of CRC. ${ }^{11,12}$

Systematic chronic inflammation could contribute to both abnormal peripheral immune cell count and aberrant concentrations of circulating albumin (Alb) and pre-albumin (pre-Alb) as well as fibrinogen (Fib). Our preview study indicated that preoperative neutrophil-to-lymphocyte ratio was an independent prognostic factor for poor recurrence-free survival and overall survival (OS) in CRC. ${ }^{13}$ Several studies showed that elevated pretreatment plasma Fib was associated with short progression-free survival in various malignancies, including CRC. ${ }^{14,15}$ Moreover, Alb and pre-Alb were observed to be significantly lower in cancer individuals in comparison with the healthy controls. ${ }^{16,17}$ Hence, we hypothesized that the two new biomarkers, Alb-to-Fib ratio (AFR) and Fib-topre-Alb ratio (FPR), might reflect the severity of systemic inflammation and predict the survival of CRC individuals.

In this study, we measured the preoperative circulating levels of Fib, Alb, pre-Alb, carcinoembryonic antigen (CEA), and carbohydrate antigen 19-9 (CA199) and performed 3 years' follow-up to evaluate potential prognostic roles of them in 702 stage I-III surgically resected CRC individuals. Furthermore, we established and compared the prognostic efficacy of CEA-CA199-FPR (CCF), CEA-CA199 (CCI), and CEA-FPR (CFI) scores. Finally, we evaluated the roles of FPR, CEA, CA199, and CCF in predicting clinical efficacy of adjuvant chemotherapy among stage II-III CRC patients.

\section{Materials and methods Population}

In our study, 702 stage I-III CRC individuals were included. All of them were first diagnosed, and pathologically confirmed according to the seventh edition of TNM/The Union for International Cancer Control (UICC)/American Joint Committee on Cancer (AJCC) classification, and were without hematologic, hepatic, autoimmune diseases, recent infection, or other malignancies, and were from the Second Affiliated Hospital of Nanchang University and Nanjing First Hospital between January 2008 and December 2013. All the enrolled patients had only radical resection performed, without preoperative neoadjuvant radiochemotherapy, and the clinical characteristics and follow-up data were obtained from each patient. To compare the difference of FPR in recurrent/distant metastatic CRC patients and in the progression-free patients, the controls were randomly selected to match the recurrent or distant metastatic cases with gender and age in the ratio of $1: 1$, and all of them were from the progression-free patients in the follow-up period. Written informed consents were signed by each eligible patient, and the study was approved by the institutional ethic commissions of the Second Affiliated Hospital of Nanchang University and Nanjing First Hospital.

\section{Data collection and laboratory detection}

We collected clinical baseline characteristics of all the patients from medical records. All preoperative sodium citrate anticoagulant peripheral blood and serum samples were collected at 7:30-9:30 am in the period before the intervention. Clauss method was selected to detect circulating Fib using SYSMEX CA-7000 machine (Sysmex, Tokyo, Japan), and inter- and intrabatch coefficients of variation (CVs) of the kit were $<4.41 \%$ and $3.66 \%$, respectively. Bromocresol green and immune turbidimetric methods were used to measure serum Alb and pre-Alb using OLYMPUS AU5400 machine (Beckman Coulter, Tokyo, Japan), and the inter- and intrabatch CVs of the kits were $<3.17 \%$ and $1.83 \%$, and $3.09 \%$ and $2.76 \%$, respectively. Electrochemiluminescence immunoassay was used to detect CEA and CA199 by a machine from Siemens ADVIA Centaur CP (Siemens, Erlangen, Germany), and the respective inter- and intrabatch $\mathrm{CVs}$ of the kits were $<3.32 \%$ and $3.25 \%$, and $3.48 \%$ and $3.26 \%$.

\section{Follow-up}

We performed 3 years of follow-up regularly, by means of telephone, email, and medical record, every 3 months for the first 2 years and every 6 months in the third year. The deadline of the follow-up was December 2016. OS was the end point of the study, and it was calculated from the time of surgical resection to death or the deadline.

\section{Construction of novel prognostic score}

To further investigate the prognostic values of FPR, CEA, and CA199, we established and compared the CCI, CFI, and CCF scores. The CCI score was composed of CEA and CA199, and the patient with both CEA $>5 \mathrm{ng} / \mathrm{mL}$ and CA199 >37 $\mathrm{U} / \mathrm{mL}$ was given a score of 2 , and patients with only one or neither of these abnormalities were allocated a score of 1 or 0 , respectively. Similarly, the patient with both elevated CEA $(>5 \mathrm{ng} / \mathrm{mL})$ and FPR $(>18.3)$ was allocated a CFI score of 2 , and patients with only one or neither of these elevated levels were allocated a score of 1 or 0 , respectively. Additionally, CCF score consisted of preoperative FPR, CEA, and CA199, and patients with either none, one, two, or three elevated levels of them (CEA $>5 \mathrm{ng} / \mathrm{ml}, \mathrm{CA} 199>37 \mathrm{U} / \mathrm{ml}, \mathrm{FPR}>18.3)$ were considered as $0,1,2$, and 3 score, respectively.

\section{Statistics}

The optimal cutoff points of FPR, AFR, Fib, and Alb were determined by X-tile software version 3.6.1 (Yale University, 
New Haven, CT, USA), and were based on 3 years' OS. Chi-square test or Fisher's exact test were used to compare the categorical variable in the groups, and Mann-Whitney $U$ or Student's $t$-test were selected to examine the difference in continuous variables. The sample power was evaluated using PASS version 11.0.10 program (NCSS, California, USA). The difference in survival rate was calculated using Kaplan-Meier curve with log-rank test, and the independent prognostic predictor was identified by Cox proportional hazards model. The predicted efficacy of the prognostic predictors was assessed by time-dependent receiver operating characteristics (ROC) analysis. We established a 3 years' OS nomogram using R 3.3.2 software (Institute for Statistics and Mathematics, Vienna, Austria), and the predictive accuracy was evaluated by Harrell's concordance index (c-index). A $p$-value $<0.05$ was recognized as statistical significance, and all the statistical analyses were conducted using IBM SPSS 20.0 software (IBM Corporation, Armonk, NY, USA).

\section{Results}

\section{Baseline characteristics of patients}

The baseline characteristics of CRC patients in the present study are summarized in Table S1. A total of 702 patients were recruited in our study, and the powers of the sample size were 0.99 for FPR, 0.89 for AFR, 0.93 for Fib, 0.98 for pre-Alb, and 0.85 for Alb. The majority of included patients were stage II (44\%) and stage III (43\%) cases, and proportions of the cases with T1-T2 and T3-T4 depth were $15.2 \%$ and $84.8 \%$, respectively. Lymph-node metastasis was observed in 302 patients, and $89.3 \%$ of the patients were G1-G2 grade cases. Moreover, $66.1 \%$ of the eligible patients received adjuvant chemotherapy. Three hundred twenty-three (62.0\%), 152 (29.2\%), and 46 (8.8\%) patients were allocated CCI score 0,1 , and 2 , respectively. Two hundred twenty-eight (43.6\%),
$209(40.0 \%)$, and $86(16.4 \%)$ patients harbored CFI 0, 1, and 2 score, respectively. The respective numbers of patients with CCF score 0, 1, 2, and 3 were 210 (40.3\%), 190 (36.5\%), 97 (18.6\%), and 24 (4.6\%). Up to follow-up deadline, 123 patients had died and 128 patients had confirmed recurrence or distant metastasis in our study.

\section{Correlation of FPR,AFR, Fib, Alb, and pre-Alb with clinical characteristics}

Using X-tile software, we found that the optimal cutoff points based on OS were $3.8 \mathrm{~g} / \mathrm{L}$ for Fib, $33.3 \mathrm{~g} / \mathrm{L}$ for Alb, 187.4 $\mathrm{mg} / \mathrm{L}$ for pre-Alb, 18.3 for FPR, and 9.2 for AFR (Figure 1 and Figure S1). The relationships between the biomarkers and clinical characteristics are described in Table 1. All of the biomarkers were closely associated with age $>60$ years, large tumor size, and poor OS. FPR, AFR, and Fib were significantly associated with tumor size, depth of invasion, and node metastasis. Furthermore, circulating FPR in patients with stage III-IV, T3-T4 depth, N1-N2 metastasis, and tumor size $>5 \mathrm{~cm}$ was higher than in those with stage I-II, T1-T2 depth, N0 metastasis, and tumor size $\leq 5 \mathrm{~cm}$, respectively (Figure 2, Table S2). Besides, we compared the difference in circulating FPR in 128 randomly selected nonrecurrent/metastatic patients and 128 recurrent/metastatic patients. Intriguingly, FPR in recurrent patients was significantly higher than that of nonrecurrent/metastatic patients $(p<0.05)$.

\section{Prognostic roles of FPR,AFR, Fib, Alb, and pre-Alb in CRC}

In the present study, the Kaplan-Meier curve with log-rank test and Cox proportion regression model were selected to investigate the prognostic roles of the baseline characteristics and FPR, AFR, Fib, Alb, and pre-Alb in CRC. As shown in Figure 3, FPR (>18.3), AFR ( $\leq 9.2)$, Fib ( $>3.8 \mathrm{~g} / \mathrm{L})$, Alb
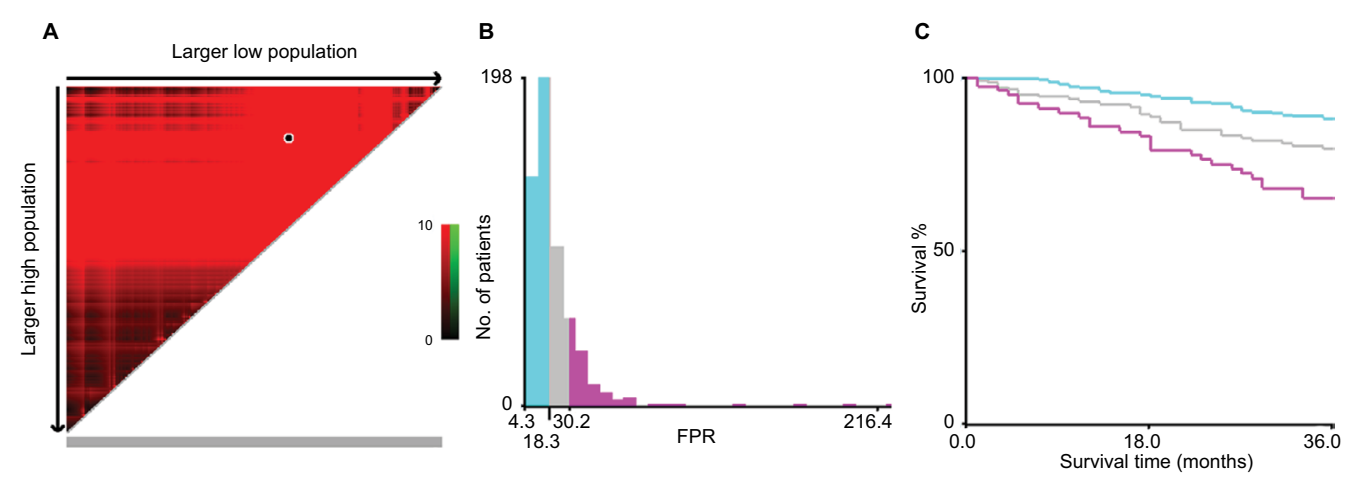

Figure I The optimal cutoff value of preoperative circulating FPR in 702 CRC patients using X-tile software.

Notes: The data are represented graphically in a right-triangular grid where the point represents the data from a given set of divisions $(\mathbf{A})$. The plots showed the $\chi^{2}$ log-rank values produced, dividing them into three groups by the cutoff points 18.3 and 30.2 . The optimal cutoff point, 18.3, was determined by locating the brightest pixel on the X-tile plot. The distribution of number of patients is shown on the histogram (B) and the corresponding populations are displayed on the Kaplan-Meier curve (C). Larger low population= larger population with low FPR level; larger high population= larger population with high FPR level.

Abbreviations: CRC, colorectal cancer; FPR, fibrinogen-to-pre-albumin ratio. 


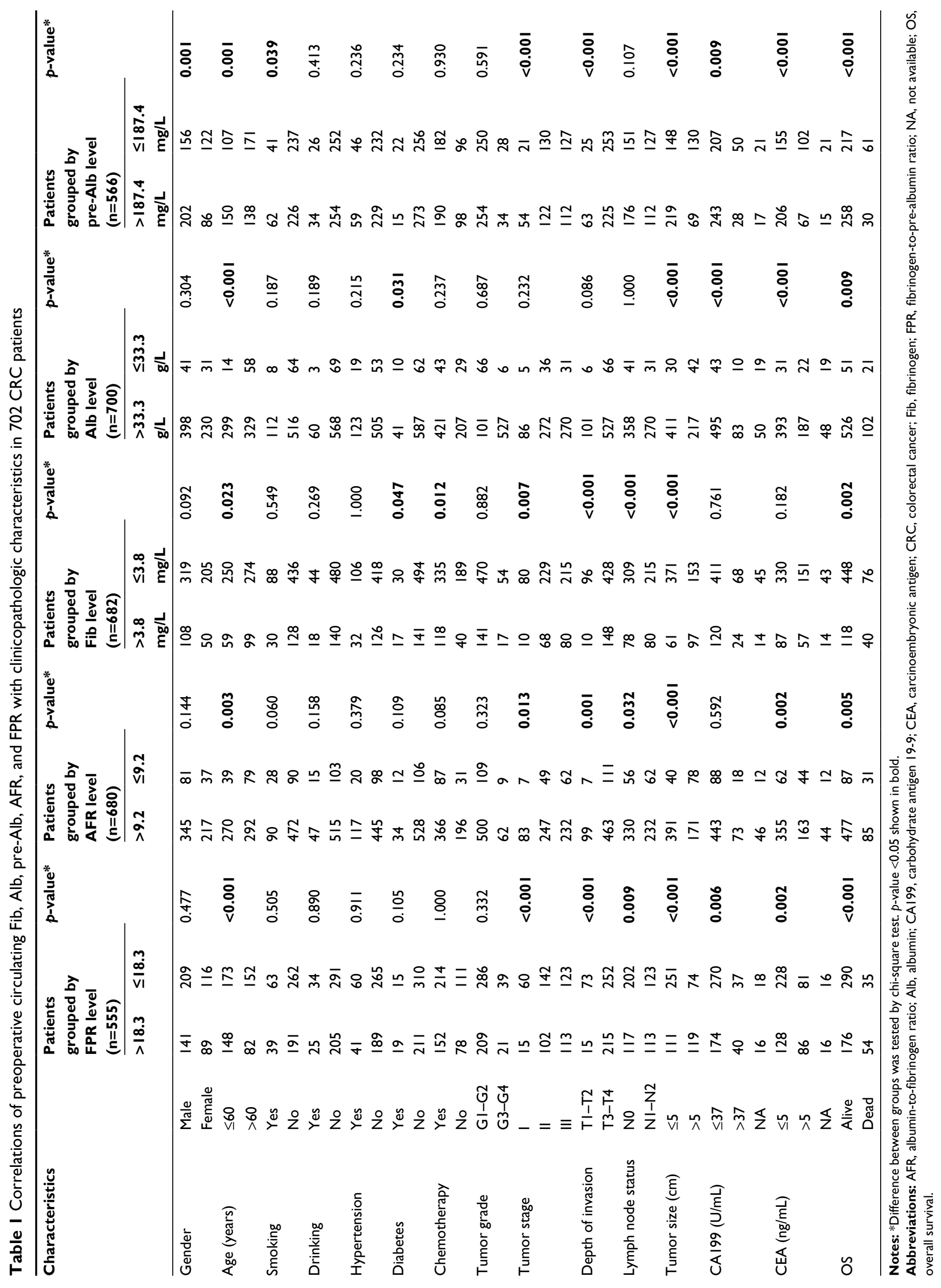



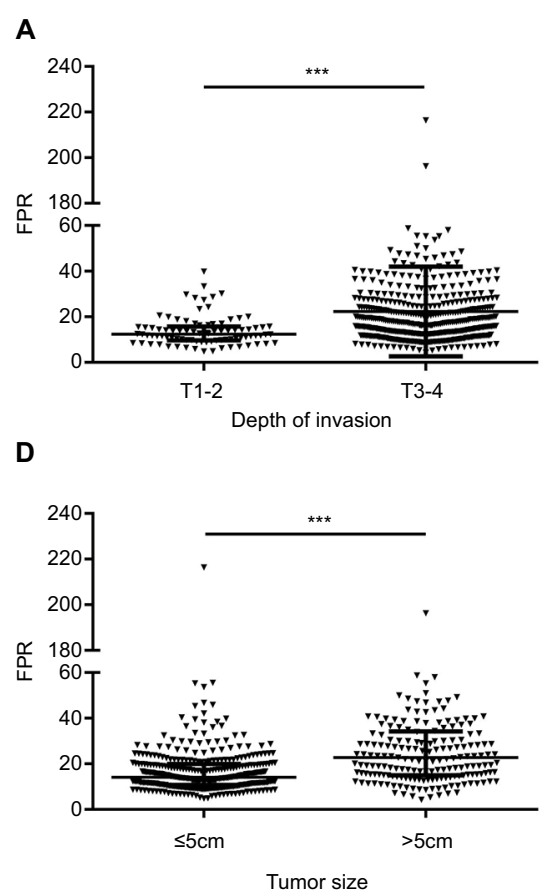
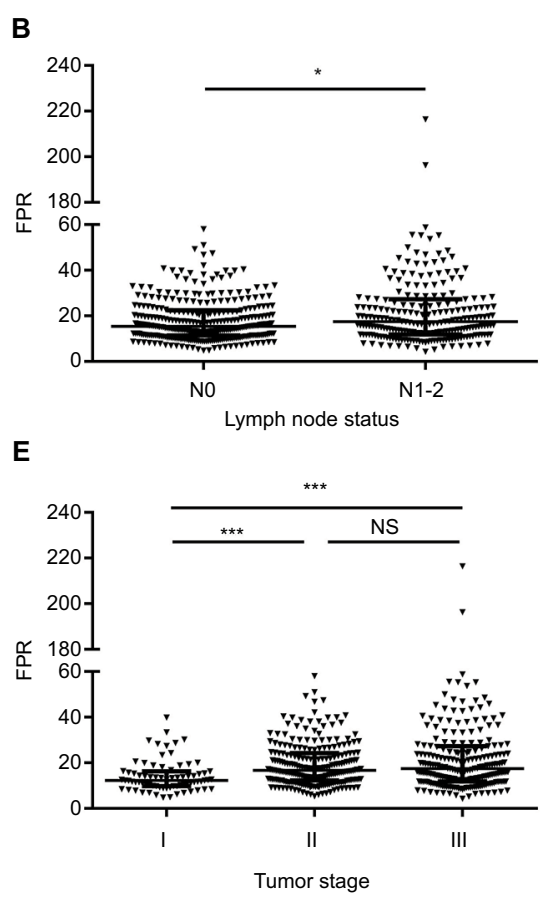

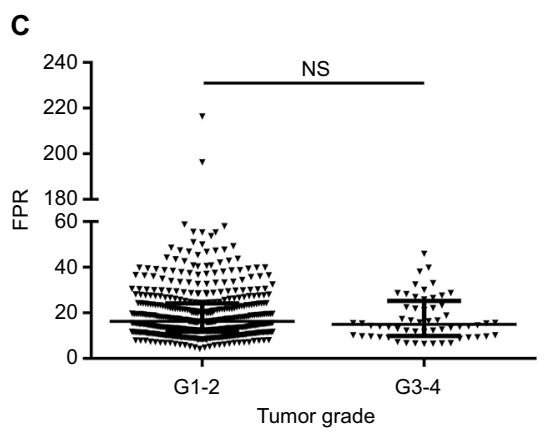

$\mathbf{F}$

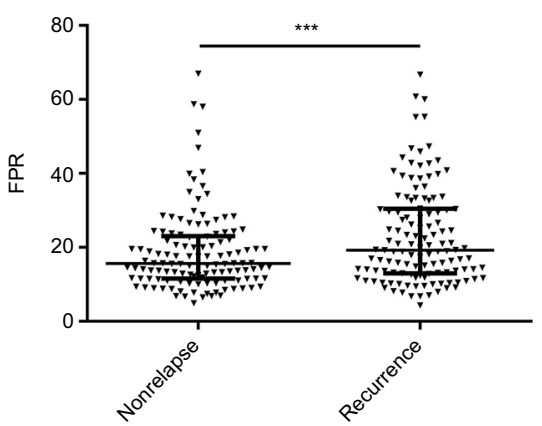

Figure 2 The correlation of FPR with clinical characteristics and comparison of FPR in nonrelapse and recurrent CRC patients.

Notes: (A) FPR with depth of invasion; (B) FPR with lymph node; (C) FPR with tumor grade; (D) FPR with tumor size; (E) FPR with tumor stage; (F) comparison of FPR in nonrelapse and recurrent CRC patients. ${ }^{*} p<0.05$, ${ }^{*} p<0.01$, *** $p<0.001$.

Abbreviations: CRC, colorectal cancer; FPR, fibrinogen-to-pre-albumin ratio; NS, no significance.

$(\leq 33.3 \mathrm{~g} / \mathrm{L})$, and pre-Alb $(\leq 187.4 \mathrm{mg} / \mathrm{L})$ were negatively associated with 3 years' OS in the Kaplan-Meier curve. G3-G4 differentiation (crude hazard ratio $[\mathrm{HR}]=1.867$, 95\% CI $=1.145-3.046$; adjusted $\mathrm{HR}=1.718,95 \% \mathrm{CI}=1.052$ 2.805), stage III (crude $\mathrm{HR}=3.834,95 \% \mathrm{CI}=1.567-9.381$; adjusted $\mathrm{HR}=3.368,95 \% \mathrm{CI}=1.370-8.280)$, $\mathrm{T} 3-\mathrm{T} 4$ depth (crude $\mathrm{HR}=3.792,95 \% \mathrm{CI}=1.669-8.614$; adjusted $\mathrm{HR}=2.792,95 \% \mathrm{CI}=1.208-6.452$ ), lymph-node metastasis (crude $\mathrm{HR}=1.934,95 \% \mathrm{CI}=1.354-2.762$; adjusted $\mathrm{HR}=1.888,95 \% \mathrm{CI}=1.322-2.698)$, large tumor size $(>5$ $\mathrm{cm}$ ) (crude $\mathrm{HR}=1.979,95 \% \mathrm{CI}=1.389-2.819$; adjusted $\mathrm{HR}=1.905,95 \% \mathrm{CI}=1.336-2.715)$, high CEA $(>5 \mathrm{ng} / \mathrm{mL}$; crude $\mathrm{HR}=2.961,95 \% \mathrm{CI}=2.019-4.342$; adjusted $\mathrm{HR}=2.719$, 95\% CI=1.850-3.996), and CA199 (>37 U/mL; crude $\mathrm{HR}=2.916,95 \% \mathrm{CI}=1.934-4.398$; adjusted $\mathrm{HR}=2.605$, $95 \% \mathrm{CI}=1.683-4.033)$ were significantly correlated with poor OS (Table 2).

Circulating elevated FPR (crude HR=2.398, 95\% $\mathrm{CI}=1.567-3.669$; adjusted $\mathrm{HR}=1.940,95 \% \mathrm{CI}=1.236-3.046$ ) was significantly associated with an increased death risk from CRC, whereas high Alb (crude $\mathrm{HR}=0.504$, 95\% $\mathrm{CI}=0.315-0.806$; adjusted $\mathrm{HR}=0.564,95 \% \mathrm{CI}=0.349-0.912$ ) and pre-Alb (crude HR=0.444, 95\% CI $=0.287-0.687$; adjusted $\mathrm{HR}=0.520,95 \% \mathrm{CI}=0.329-0.823$ ) were significantly associated with decreased death risk from the disease.
However, no correlations were observed between AFR, Fib, and OS (Table 2).

\section{Analysis of time-dependent ROC}

Time-dependent ROC was used to compare the prognostic efficacy of each biomarker in our study. The areas under the ROC (AUCs) of FPR, Alb, and pre-Alb were larger than for the other biomarkers in the first few months (Figure 3I). Comparatively, AUC of FPR stayed at the higher level compared to Alb and pre-Alb, in the following months. In addition, CEA had the largest AUC in the last 24 months.

\section{Prognostic values of $\mathrm{CCl}, \mathrm{CFI}$, and $\mathrm{CCF}$ scores}

To further investigate the prognostic values of FPR, CEA, and CA199, we established and assessed the prognostic roles of CCI, CFI, and CCF scores. The higher CCI (crude HR=2.495 and adjusted $\mathrm{HR}=2.289$ for score 1 ; crude $\mathrm{HR}=5.926$ and adjusted $\mathrm{HR}=5.612$ for score 2), $\mathrm{CFI}$ (crude $\mathrm{HR}=3.021$ and adjusted $\mathrm{HR}=2.748$ for score 1 ; crude $\mathrm{HR}=6.957$ and adjusted $\mathrm{HR}=5.904$ for score 2), and CCF (crude HR=2.770 and adjusted $\mathrm{HR}=2.578$ for score 1 ; crude $\mathrm{HR}=5.902$ and adjusted $\mathrm{HR}=4.753$ for score 2; crude $\mathrm{HR}=12.082$ and adjusted $\mathrm{HR}=11.518$ for score 3 ) were significantly associated with reduced survival of the CRC patients (Figure 4 and Table $\mathrm{S} 3$ ). 
A

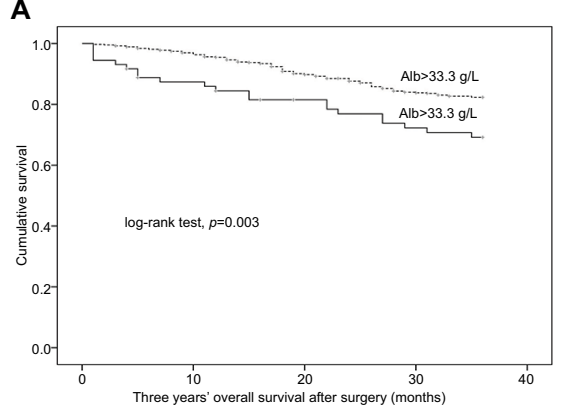

D

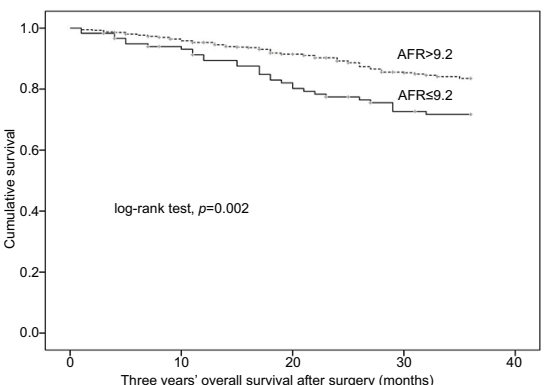

G

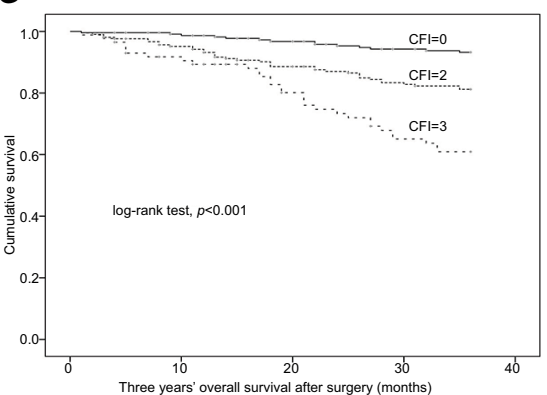

B

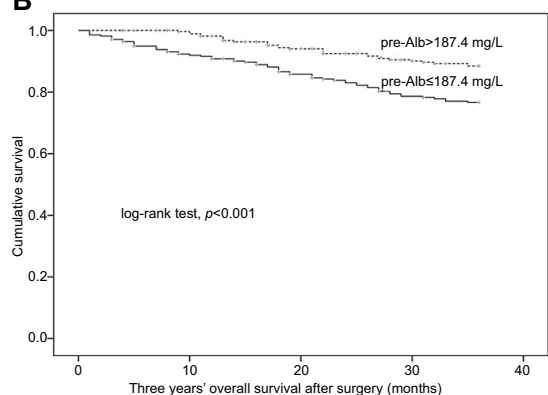

E

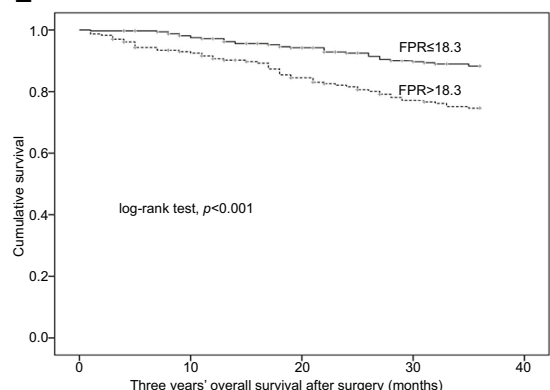

H

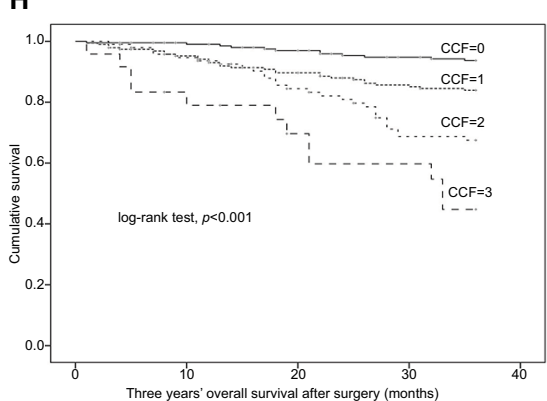

C

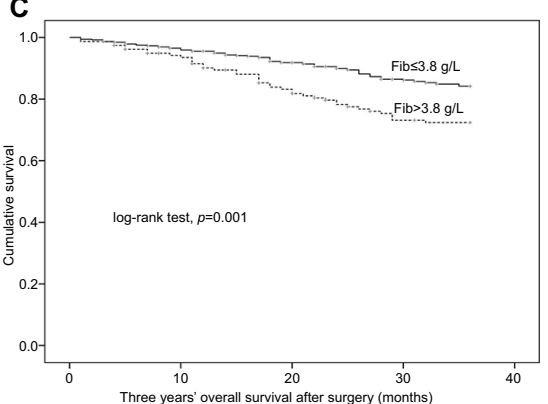

F

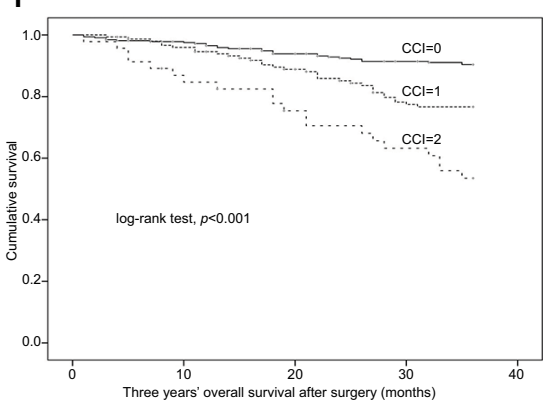

I

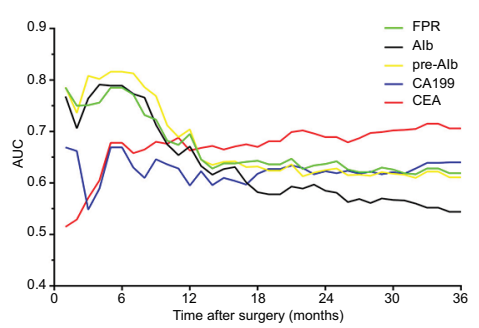

Figure 3 Kaplan-Meier curves of FPR, AFR, Fib, Alb, and pre-Alb for 3 years' OS in 702 CRC patients.

Notes: (A) Alb; (B) pre-Alb; (C) Fib; (D) AFR; (E) FPR; (F) CCl; (G) CFI; (H) CCF. Time-dependent receiver operating characteristic analysis of preoperative circulating FPR, Alb, pre-Alb, CA 199, and CEA for clinical outcome of 702 CRC patients (I).

Abbreviations: AFR, albumin-to-fibrinogen ratio; Alb, albumin; pre-Alb, pre-albumin; CA199, carbohydrate antigen 19-9; CCF, CEA-CAI99-FPR; CCI, CEA-CAI99; CEA, carcinoembryonic antigen; CFI, CEA-FPR; CRC, colorectal cancer; Fib, fibrinogen; FPR, fibrinogen-to-pre-albumin ratio; HR, hazard ratio; OS, overall survival.

\section{Predictive roles of FPR, CEA, CA I99, and CCF in chemotherapy}

To investigate the predictive roles of FPR, CEA, CA199, and CCF in adjuvant chemotherapy, we compared the survival difference of surgical stage II-III CRC patients with or without treatment of chemotherapy in each high or low FPR, CEA, CA199, and CCF subgroup. We found that OS of the patients with adjuvant chemotherapy was significantly longer than in those without it, only in $\mathrm{CCF} \geq 1$ score $(p<0.05$, adjusted $\mathrm{HR}=0.494,95 \% \mathrm{CI}=0.303-0.806)$ and high FPR $(p<0.05$, adjusted $\mathrm{HR}=0.420,95 \% \mathrm{CI}=0.241-0.731)$ subgroups. However, no survival difference was observed in the low FPR and CCF score subgroups, or in all subgroups stratified by CEA and CA199 (Figure 5 and Figure S2).

\section{Assessment of the constructed nomograms}

The pivotal clinical pathologic characteristics, FPR, and CCF scores were selected to construct prognostic nomograms to predict 3 years' OS of CRC and the predicted accuracy was evaluated by Harrell's c-index. The nomograms are listed in Figure 6 and Figure S3; c-indexes of the nomograms with or without CCF score were 0.728 (0.677-0.777) and $0.626(0.568-0.684)$, and c-indexes of those with or without FPR were 0.722 (0.667-0.777) and $0.706(0.648-0.764)$, respectively. Moreover, the significant difference between c-indexs of the nomogram with or without CCF score was observed between them $(p<0.01)$. 
Table 2 Univariate and multivariate analyses of prognostic factors for 3-year overall survival by Cox regression model

\begin{tabular}{|c|c|c|c|c|}
\hline \multirow[t]{3}{*}{ Variables } & \multicolumn{4}{|l|}{ Overall survival } \\
\hline & \multicolumn{2}{|c|}{ Univariate cox regression } & \multicolumn{2}{|c|}{ Multivariate cox regression } \\
\hline & Crude HR (95\% Cl) & $p$-value & Adjusted HR (95\% Cl) & $p$-value \\
\hline Sex (female) & $0.880(0.606-1.277)$ & 0.501 & - & - \\
\hline Age (>60 years) & I.223 (0.85I-2.805) & 0.277 & - & - \\
\hline Alcohol (yes) & $1.186(0.654-2.152)$ & 0.575 & - & - \\
\hline Tobacco (yes) & $0.995(0.622-1.591)$ & 0.982 & - & - \\
\hline Hypertension (yes) & $0.878(0.558-1.382)$ & 0.575 & - & - \\
\hline Diabetes (yes) & $1.115(0.584-2.130)$ & $0.74 I$ & - & - \\
\hline Chemotherapy (yes) & $0.882(0.6 \mathrm{II}-1.274)$ & 0.504 & - & - \\
\hline Tumor grade (G3-G4) & $1.867(1.145-3.046)$ & 0.012 & $1.718(1.052-2.805)$ & 0.030 \\
\hline Tumor stage (III) & $3.834(1.567-9.38 I)$ & 0.003 & $3.368(1.370-8.280)$ & 0.008 \\
\hline Depth of invasion (T3-T4) & $3.792(1.669-8.614)$ & 0.001 & $2.792(1.208-6.452)$ & 0.016 \\
\hline lymph node (NI-N3) & $1.934(1.354-2.762)$ & $<0.001$ & $1.888(1.322-2.698)$ & $<0.001$ \\
\hline Tumor size $(>5 \mathrm{~cm})$ & $1.979(1.389-2.819)$ & $<0.001$ & $1.905(1.336-2.715)$ & 0.001 \\
\hline CEA $(>5 \mathrm{ng} / \mathrm{mL})$ & $2.961(2.019-4.342)$ & $<0.001$ & $2.719(1.850-3.996)$ & $<0.001$ \\
\hline CAI 99 (>37 U/mL) & $2.916(1.934-4.398)$ & $<0.001$ & $2.605(1.683-4.033)$ & $<0.001$ \\
\hline Fib $(>3.8 \mathrm{mg} / \mathrm{dL})$ & $1.913(1.304-2.806)$ & 0.001 & $1.450(0.96 \mathrm{I}-2.188)$ & 0.077 \\
\hline Alb $(>33.3 \mathrm{~g} / \mathrm{L})$ & $0.504(0.3 \mid 5-0.806)$ & 0.004 & $0.564(0.349-0.912)$ & 0.020 \\
\hline pre-Alb (>I87.4 mg/L) & $0.444(0.287-0.687)$ & $<0.001$ & $0.520(0.329-0.823)$ & 0.005 \\
\hline $\operatorname{AFR}(>9.2)$ & $0.53 \mathrm{I}(0.352-0.80 \mathrm{I})$ & 0.003 & $0.709(0.455-1.107)$ & 0.130 \\
\hline FPR $(>18.3)$ & $2.398(1.567-3.669)$ & $<0.001$ & $1.940(1.236-3.046)$ & 0.004 \\
\hline
\end{tabular}

Notes: Adjusted HR (95\%) was adjusted by sex, age, alcohol, tobacco, hypertension, diabetes, chemotherapy, tumor size, tumor grade, and tumor stage. The endash represent that these variables were not included in the multivariate cox regression. $p$-value $<0.05$ shown in bold.

Abbreviations: AFR, albumin/fibrinogen ratio; Alb, albumin; pre-Alb, pre-albumin; CA199, carbohydrate antigen 19-9; CEA, carcinoembryonic antigen; Fib, fibrinogen; FPR, fibrinogen-to-pre-albumin ratio; HR, hazard ratio.

A

Cox regression analysis

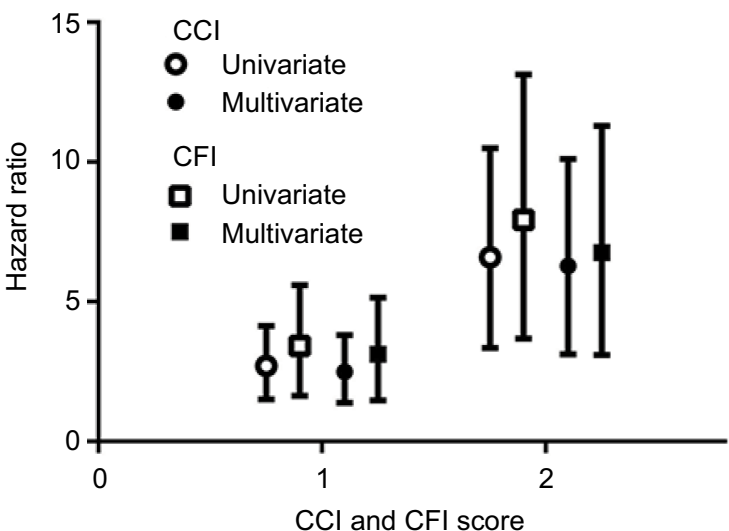

B

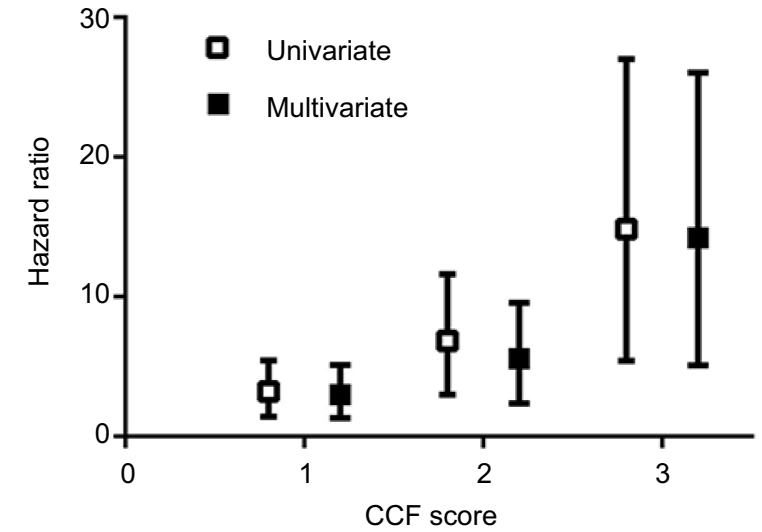

Figure 4 The HRs of $\mathrm{CCl}, \mathrm{CFI}$, and CCF scores in CRC patients.

Notes: (A) The crude and adjusted HRs of $\mathrm{CCl}$ and CFI score; (B) The crude and adjusted HRs of CCF score.

Abbreviations: CCF, CEA-CAI99-FPR; CCl, CEA-CAI99; CAI99, carbohydrate antigen 19-9; CEA, carcinoembryonic antigen; CFI, CEA-FPR; CRC, colorectal cancer; $H R$, hazard ratio; FPR, fibrinogen-to-pre-albumin ratio.

\section{Discussion}

Systemic inflammation is one of the important hallmarks of CRC. ${ }^{7}$ CRC cells, cancer stem cells, chronic inflammatory mediators, and inflammation-related cells, such as cancer-associated fibroblasts and endothelial cells, as well as tumor-associated macrophages form a favorable microenvironment to promote carcinogenesis of colorectal epithelial cells and to create a pre-metastatic niche in secondary organs or tissue sites for subsequent metastasis. ${ }^{18-20}$
Circulating Fib, Alb, and pre-Alb were the main inflammatory biomarkers, and hyperfibrinogenemia and hypoalbuminemia were commonly observed in CRC patients. ${ }^{21,22}$ However, the prognostic and predictive roles of AFR and FPR in surgically resected CRC remained unknown.

We conducted this prospective study to investigate the association of preoperative AFR, FPR, Fib, Alb, pre-Alb, CEA, and CA199 with 3 years' OS in 702 I-III surgically resected CRC individuals. Our results showed that low AFR, Alb, pre-Alb, 

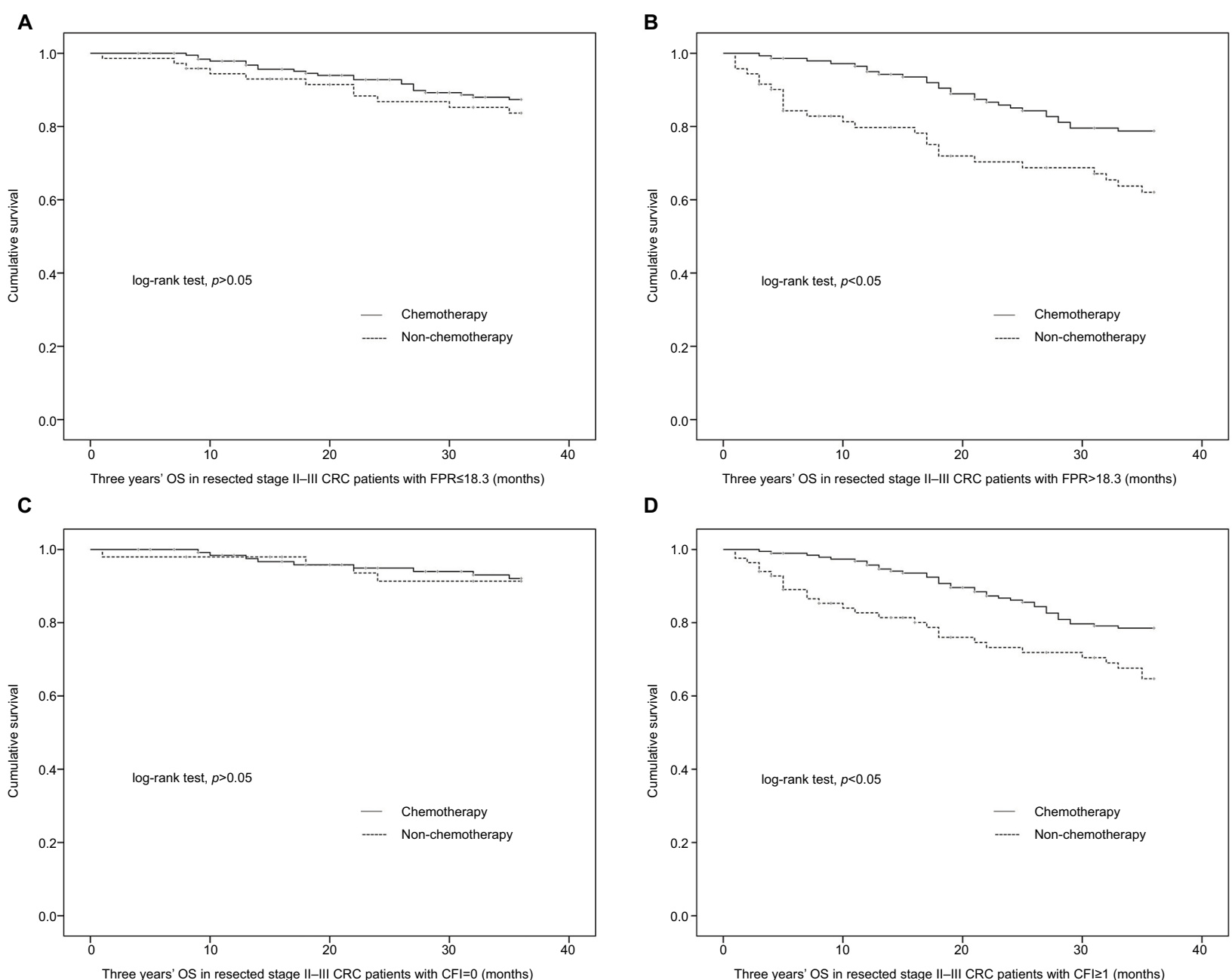

D
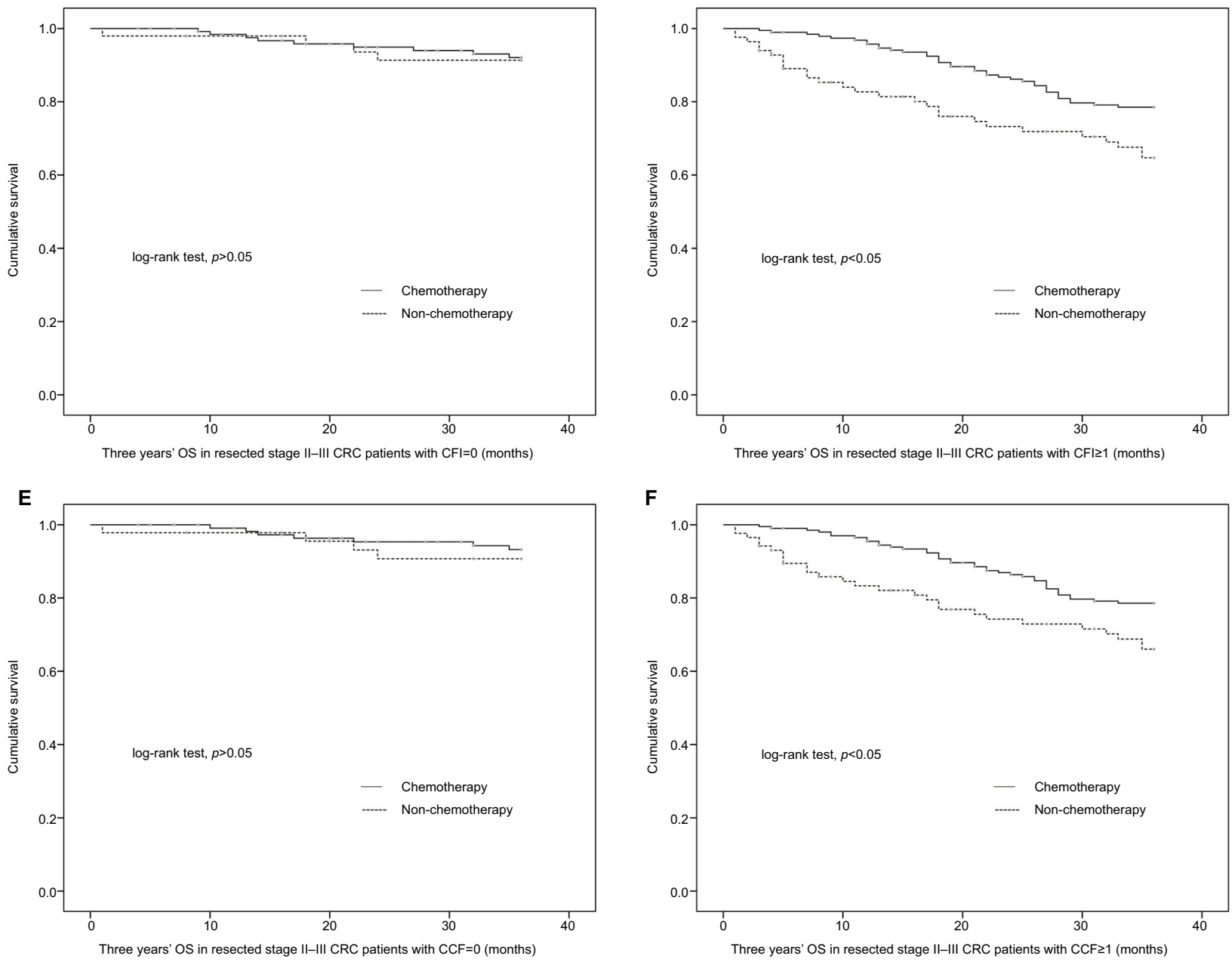

Figure 5 Kaplan-Meier curves of stage II-III CRC patients with or without treatment of chemotherapy in each subgroup stratified by FPR, CFI, and CCF. Notes: (A) FPR $\leq 18.3$; (B) FPR $>18.3$; (C) $C F I=0$; (D) $C F I \geq 1$; (E) $C C F=0$; (F) $C C F \geq 1$.

Abbreviations: CCF, CEA-CAI99-FPR; CFI, CEA-FPR; CRC, colorectal cancer; FPR, fibrinogen-to-pre-albumin ratio; OS, overall survival.

and elevated Fib level were only associated with T3-T4 invasion and tumor size. However, elevated FPR was significantly associated with TNM stage, invasion depth, node metastasis, and tumor size. These results indicated that FPR was superior to these biomarkers to in evaluating CRC progression and the tumor burden. Furthermore, low Alb and pre-Alb, and high FPR were significantly associated with poor survival of CRC, and the predicted efficacy of FPR was significantly higher than the 
A

Points

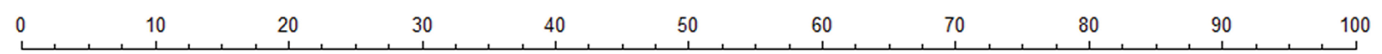

Tumor stage

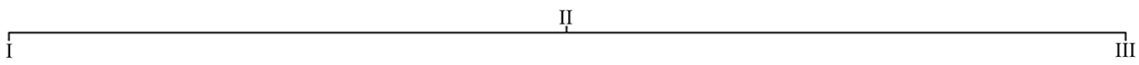

Differentiation

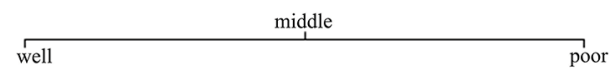

Size $(\mathrm{cm})$

Depth of invasion

Node metastasis

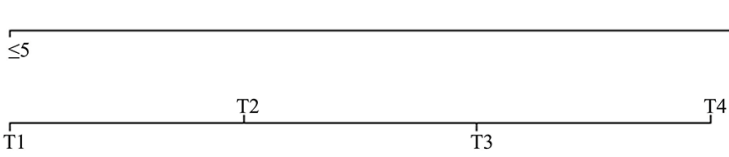

Total points

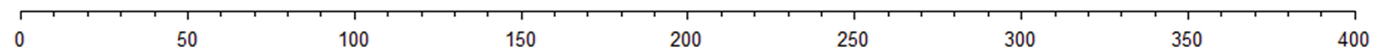

3-year mortality risk

\begin{tabular}{lllll}
\hline 0.3 & 1 & 1 & \\
& 0.4 & 0.5 & 0.6 & 0.7
\end{tabular}

B

Points

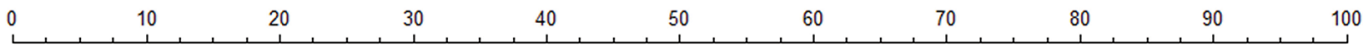

Tumor stage

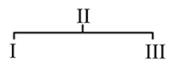

Differentiation

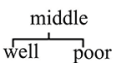

Size $(\mathrm{cm})$

Depth of invasion

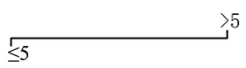

Node metastasis

$\stackrel{\mathrm{T} 2}{\mathrm{~T} 1} \stackrel{\mathrm{T} 3}{\mathrm{~T} 3}$

$\mathrm{CCF}$

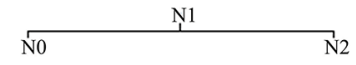

Total points
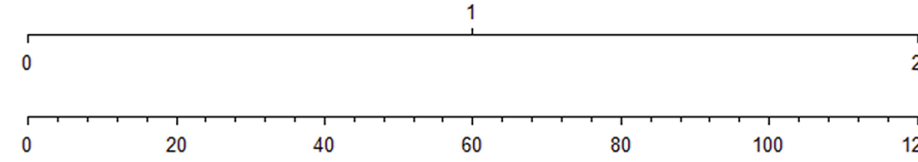

$20 \quad 40$

$40 \quad 60$

$80 \quad 100$

120

140

160

180

3-year mortality risk

0.3

0.4

0.5

0.7

0.8

0.9

Figure 6 Prognostic nomograms with or without CCF score for predicting 3-year OS in CRC patients.

Notes: (A) Nomogram without CCF; (B) Nomogram including CCF.

Abbreviations: CCF, CEA-CAI99-FPR; CRC, colorectal cancer; FPR, fibrinogen-to-pre-albumin ratio; OS, overall survival.

others, demonstrating that it was an effective and independent prognostic factor to predict the prognosis of CRC.

In addition to the single inflammatory biomarker, the emerging novel prognostic scores have been proposed to evaluate the progression and survival of CRC. Glasgow prognostic score (GPS) and modified GPS showed good performance in predicting clinical outcome of CRC. ${ }^{23,24}$ Depending on FPR, CEA, and CA199, three novel CFI,
CCI, and CCF scores were first established in our study, and they were obviously associated with the survival of CRC; however, the measured HRs of CCI and CFI score were less than CCF score, suggesting that the two scores were inferior to $\mathrm{CCF}$ to predict survival of CRC. Moreover, no significant survival difference of stage II-III CRC patients with or without adjuvant chemotherapy was observed in any subgroup stratified by CEA and CA199, whereas the 
cases that harbored high FPR and CCF score could benefit from adjuvant chemotherapy, illustrating that FPR and CCF score were useful biomarkers to precisely distinguish eligible patients who could benefit from the treatment. The c-index of the nomogram containing FPR or CCF was significantly higher than the nomogram without them, suggesting that FPR and CCF could improve predicted accuracy of the prognostic nomogram.

Notably, Fib is not only an important factor in the blood coagulation cascade but also a main acute phase reaction protein in chronic inflammation. Tumor cells could interact with all parts of the hemostatic system to stimulate prothrombotic properties and to trigger the production of Fib by liver and by itself, ${ }^{25}$ contributing to the high level of Fib. Meanwhile, Fib was considered as a mediator of cancer cell proliferation; ${ }^{26}$ it sustained the adhesion and survival of cancer cell emboli in the vasculature of target organs to promote metastasis, ${ }^{27}$ and it could protect cancer cells escaping elimination by natural killer cells by means of formation of a shielding cover around them. ${ }^{28}$ Moreover, the common inflammatory cytokine, interleukin-6, was reported to suppress the synthesis of Alb and pre-Alb, leading to hypoproteinemia in CRC patients. ${ }^{29}$ Consequently, poor nutritional status and impaired immunologic surveillance of the patient directly affected clinical outcome of the disease..$^{29,30}$ Thus, these factors might have accounted for the findings in the present study.

Our study is the first to evaluate the prognostic roles of AFR, FPR, and CCF score in survival of CRC patients. The established nomogram containing CCF was an easy-to-use system for accurately estimating 3 years' survival of CRC patients after surgery. However, some limitations in the current study should be addressed. First, the diverse postoperative chemotherapy regimens might lead to the heterogeneous outcome of the patients. Secondly, only 3 years' OS was included in our study, and we did not obtain the complete recurrence data and 5 years' of OS, so our study can not comprehensively reflect survival of the patients. Thirdly, no validated cohort was included to verify the findings in our study.

\section{Conclusion}

In summary, our data have revealed that FPR and $\mathrm{CCF}$ score are reliable, economical, and practical biomarkers to precisely distinguish eligible patients for treatment with adjuvant chemotherapy and to predict the prognosis of CRC. Further, multicenter and large sample size design studies are warranted to validate our results.

\section{Acknowledgment}

This study was supported by the 2018 Scientific Fund of Health and Family Planning Commission of Jiangxi Province (20185250).

\section{Author contributions}

All authors contributed toward data analysis, drafting and revising the paper and agree to be accountable for all aspects of the work.

\section{Disclosure}

The authors report no conflicts of interest in this work.

\section{References}

1. Siegel RL, Miller KD, Jemal A. Cancer statistics, 2017. CA Cancer J Clin. 2017;67(1):7-30.

2. Fitzmaurice C, Dicker D, Pain A, et al. The global burden of cancer 2013. JAMA Oncol. 2015;1(4):505-527.

3. Chen W, Zheng R, Baade PD, et al. Cancer statistics in China, 2015. CA Cancer J Clin. 2016;66(2):115-132.

4. Siegel RL, Miller KD, Fedewa SA, et al. Colorectal cancer statistics, 2017. CA Cancer J Clin. 2017;67(3):177-193.

5. Balkwill F, Mantovani A. Inflammation and cancer: back to Virchow? Lancet. 2001;357(9255):539-545.

6. Mantovani A, Allavena P, Sica A, Balkwill F. Cancer-related inflammation. Nature. 2008;454(7203):436-444.

7. Terzic J, Grivennikov S, Karin E, Karin M. Inflammation and colon cancer. Gastroenterology. 2010;138(6):2101-2114.e2105.

8. Grivennikov SI. Inflammation and colorectal cancer: colitis-associated neoplasia. Semin Immunopathol. 2013;35(2):229-244.

9. Rubin DC, Shaker A, Levin MS. Chronic intestinal inflammation: inflammatory bowel disease and colitis-associated colon cancer. Front Immunol. 2012;3:107.

10. Mei Z, Liu Y, Liu C, et al. Tumour-infiltrating inflammation and prognosis in colorectal cancer: systematic review and meta-analysis. $\mathrm{Br} \mathrm{J}$ Cancer. 2014;110(6):1595-1605.

11. Friis S, Riis AH, Erichsen R, Baron JA, Sorensen HT. Low-dose aspirin or nonsteroidal anti-inflammatory drug use and colorectal cancer risk: a population-based, case-control study. Ann Intern Med. 2015;163(5):347-355.

12. Nan H, Hutter CM, Lin Y, et al. Association of aspirin and NSAID use with risk of colorectal cancer according to genetic variants. JAMA. 2015;313(11):1133-1142.

13. Ying HQ, Deng QW, He BS, et al. The prognostic value of preoperative NLR, d-NLR, PLR and LMR for predicting clinical outcome in surgical colorectal cancer patients. Med Oncol. 2014;31(12):305.

14. Perisanidis C, Psyrri A, Cohen EE, et al. Prognostic role of pretreatment plasma fibrinogen in patients with solid tumors: a systematic review and meta-analysis. Cancer Treat Rev. 2015;41(10):960-970.

15. Lee JH, Hyun JH, Kim DY, et al. The role of fibrinogen as a predictor in preoperative chemoradiation for rectal cancer. Ann Surg Oncol. 2015;22(1):209-215.

16. Gupta D, Lis CG. Pretreatment serum albumin as a predictor of cancer survival: a systematic review of the epidemiological literature. Nutr J. 2010;9:69.

17. Huang J, Wang Y, Yuan Y, et al. Preoperative serum pre-albumin as an independent prognostic indicator in patients with localized upper tract urothelial carcinoma after radical nephroureterectomy. Oncotarget. 2017;8(22):36772-36779.

18. Pollard JW. Tumour-educated macrophages promote tumour progression and metastasis. Nat Rev Cancer. 2004;4(1):71-78. 
19. Pages F, Berger A, Camus M, et al. Effector memory T cells, early metastasis, and survival in colorectal cancer. $N$ Engl J Med. 2005;353(25): 2654-2666.

20. Wolf D, Marth C, Wolf AM. Immune cells in colorectal cancer. N Engl J Med. 2006;354(14):1531-1532; author reply 1531-1532.

21. Son HJ, Park JW, Chang HJ, et al. Preoperative plasma hyperfibrinogenemia is predictive of poor prognosis in patients with nonmetastatic colon cancer. Ann Surg Oncol. 2013;20(9):2908-2913.

22. Hu WH, Cajas-Monson LC, Eisenstein S, Parry L, Cosman B, Ramamoorthy S. Preoperative malnutrition assessments as predictors of postoperative mortality and morbidity in colorectal cancer: an analysis of ACS-NSQIP. Nutr J. 2015;14:91.

23. Ishizuka M, Nagata H, Takagi K, Horie T, Kubota K. Inflammationbased prognostic score is a novel predictor of postoperative outcome in patients with colorectal cancer. Ann Surg. 2007;246(6):1047-1051.

24. McSorley ST, Black DH, Horgan PG, McMillan DC. The relationship between tumour stage, systemic inflammation, body composition and survival in patients with colorectal cancer. Clin Nutr. 2017.
25. Caine GJ, Stonelake PS, Lip GY, Kehoe ST. The hypercoagulable state of malignancy: pathogenesis and current debate. Neoplasia. 2002;4(6):465-473.

26. Simpson-Haidaris PJ, Rybarczyk B. Tumors and fibrinogen. The role of fibrinogen as an extracellular matrix protein. Ann NY Acad Sci. 2001;936:406-425.

27. Palumbo JS, Kombrinck KW, Drew AF, et al. Fibrinogen is an important determinant of the metastatic potential of circulating tumor cells. Blood. 2000;96(10):3302-3309.

28. Zheng S, Shen J, Jiao Y, et al. Platelets and fibrinogen facilitate each other in protecting tumor cells from natural killer cytotoxicity. Cancer Sci. 2009;100(5):859-865.

29. Fujii T, Sutoh T, Morita H, et al. Serum albumin is superior to prealbumin for predicting short-term recurrence in patients with operable colorectal cancer. Nutr Cancer. 2012;64(8):1169-1173.

30. Van Cutsem E, Arends J. The causes and consequences of cancerassociated malnutrition. Eur J Oncol Nurs. 2005;9(Suppl 2):S51-S63.
Cancer Management and Research

\section{Publish your work in this journal}

Cancer Management and Research is an international, peer-reviewed open access journal focusing on cancer research and the optimal use of preventative and integrated treatment interventions to achieve improved outcomes, enhanced survival and quality of life for the cancer patient. The manuscript management system is completely online and includes

\section{Dovepress}

a very quick and fair peer-review system, which is all easy to use. Visit http://www.dovepress.com/testimonials.php to read real quotes from published authors. 